\title{
Status and expected performance of the LHCb tracking system
}

Matthew Needham ${ }^{\text {a }}$

For the LHCb collaboration

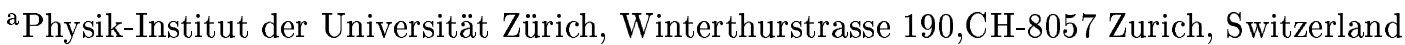

The current status of the components of the LHCb tracking system and their expected performance are described in detail.

\section{INTRODUCTION}

$\mathrm{LHCb}[1]$ is a dedicated B physics experiment that will operate at the LHC. The layout of the detector is shown in Fig. 1. It will perform precision measurements of $\mathrm{CP}$ violation and rare decays in the $B$ meson system. Efficient and precise reconstruction of the trajectories of charged particles by the tracking system is a key requirement of the experiment. The tracking system consists of a silicon vertex locator, a large area silicon station (TT), magnet and three stations located downstream of the magnet. The latter, stations T1-T3, are divided into an inner part (Inner Tracker) using silicon and an outer part (Outer Tracker) using straw tubes. In the following sections the current status of each of the subsystems is described.

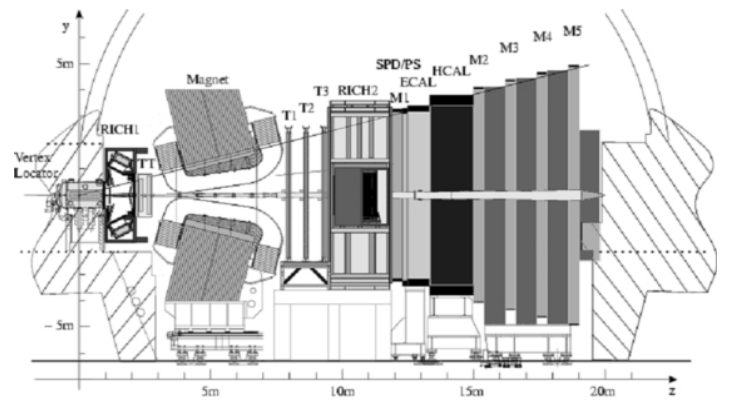

Figure 1. The LHCb detector (non-bend plane).

\subsection{Vertex Locator}

The design of the Vertex Locator (VeLo) is described in [2]. It consists of 21 stations situated around the interaction point. The detectors are installed inside the LHC vacuum vessel. A $250 \mu \mathrm{m}$ thick corrugated aluminium foil separates the detectors from the beam vacuum and acts as RF shield and wake-field suppressor for the LHC beams. The closest strips will be at a distance of $8 \mathrm{~mm}$ from the LHC beam axis. During beam injection the detectors will be retracted.

A $r-\phi$ strip geometry has been chosen for the sensors. Each station consists of two discs of silicon with an outer radius of $4.2 \mathrm{~cm}$. One disk has radial strips whilst the other has $\phi$ measuring strips. Each of these discs consists of two semi-circular silicon sensors. The layout of the two types of sensor is shown in Fig. 2. The pitch of the detector ranges from $40 \mu \mathrm{m}$ at small radii up to $100 \mu \mathrm{m}$ at the detector edge. The choice of a $\mathrm{r}-\phi$ layout allows the use of fast 2-D tracking algorithms in the L1 trigger [3].

Since the innermost strips are close to the LHC beams the radiation dose seen by the VeLo is large. Fluences as high as $1.3 \times 10^{14} 1-\mathrm{MeV}$ neutron equivalent per $\mathrm{cm}^{2}$ are expected in the innermost strips per year. A detailed R\&D program has been carried out to develop sensors that will provide adequate signal-to-noise performance for several years of $\mathrm{LHCb}$ operation after which it is forseen to replace the sensors. Based on these studies it has been chosen to use $300 \mu \mathrm{m}$ thick $\mathrm{n}$-on-n silicon.

The vacuum vessel that houses the detector has been produced and module production will start 


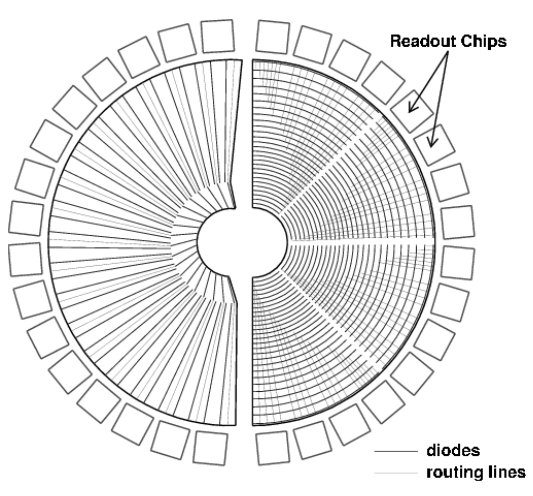

Figure 2. Layout of a Velo $\phi$ sensor (left) and $r$ sensor (right).

in the next months. A testbeam with the complete detector is foreseen at CERN in April 2006.

\subsection{Trigger Tracker}

The Trigger Tracker [4] is located in the fringe field of the magnet and covers the full detector acceptance with silicon micro-strip detectors. It will consist of four detection layers arranged into two half stations separated by $30 \mathrm{~cm}$ along the beam axis. The splitting of the station allows a first determination of the particle momentum in the Level-1 trigger. The detector will be constructed using $500 \mu \mathrm{m}$ thick p-on-n type sensors with a pitch of $183 \mu \mathrm{m}$ of the same design as used in the CMS barrel.

The layout of a detection layer is illustrated in Fig. 3 . The areas above and below the beam pipe are each covered by a single seven-sensor long silicon ladder, the areas to the left and to the right of the beam pipe are covered by 14 -sensor long ladders. Electronically, each ladder is split into several readout sectors, indicated by different shadings in Fig. 3. In the outer part of the detector, where the fluence is low the readout sectors consist of four and three sensors chained together. For the ladders closest to the beam-pipe, where the fluence is highest, the three sensor sectors are segmented into a one and a two sensor sector.

All readout electronics and associated mechan-

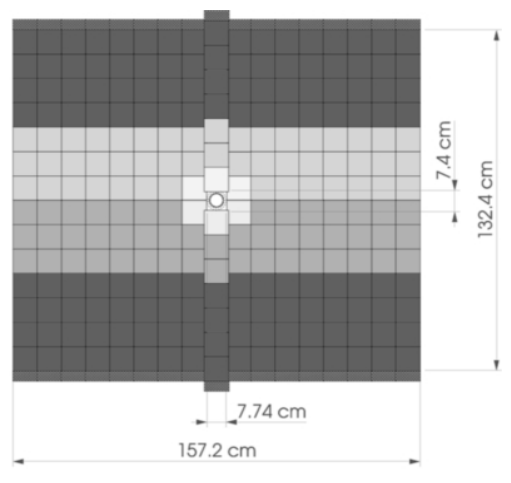

Figure 3. Layout of a layer of the Trigger Tracker. Readout sectors are indicated by the different shadings.

ics are located at the top end or the bottom end of a ladder, outside of the acceptance of the experiment. The inner readout sectors are connected to their readout electronics via Kapton interconnect cables of length $39 \mathrm{~cm}$ and $58 \mathrm{~cm}$. Capacitive loads of up to $57 \mathrm{pF}$ are seen by the front-end chip. Prototype ladders have been extensively tested and a $\mathrm{S} / \mathrm{N}$ of $\sim 15$ achieved in a $120 \mathrm{GeV}$ $\pi^{-}$beam. Production of the final modules is due to start in August 2005 and is expected to be completed by May 2006 .

\subsection{Magnet}

$\mathrm{LHCb}$ will use a warm dipole magnet whose coils follow the acceptance of $300 \times 250 \mathrm{mrad}$. The magnet gives an $\int \mathrm{Bdl}$ of $4 \mathrm{Tm}$. Assembly has been completed and the magnet installed in the experimental hall. Nominal current was achieved in the magnet during November 2004. A program to map the field is currently underway.

\subsection{Outer Tracker}

The Outer Tracker [5] covers the major part of each of the three $\mathrm{T}$ stations located after the magnet. The chosen technology is $5 \mathrm{~mm}$ diameter straw tubes with $5.25 \mathrm{~mm}$ pitch packed into double layer modules. Each station consists of four double layers in the orientation $0^{\circ}, 5^{\circ},-5^{\circ}, 0^{\circ}$ 
with respect to the vertical axis. Kapton- $\mathrm{XC}$ with an outer Aluminium winding to reduce crosstalk has been chosen as the cathode material. In order to keep the detection of spillover hits from previous bunch crossings to a low level a fast drift gas giving signal collection times of less than $50 \mathrm{~ns}$ is needed. It is intended to use $\mathrm{Ar} / \mathrm{CO}_{2}$ in the ratio $70: 30$. With this gas a maximum drift time of $\sim 40 \mathrm{~ns}$ is found in testbeam.

Construction of the Outer Tracker is well advanced. Currently, $60 \%$ of the modules have been produced. The quality of these modules is excellent. In tests using a $6 \mathrm{GeV}$ electron beam at DESY the resolution was measured to be $200 \mu \mathrm{m}$, the single cell efficiency to be $98 \%$ and the crosstalk rate to be $5 \%$. All these values are consistent with those assumed in detector performance studies. In addition, prototype support frames have been produced and a mock-up of a station has been set-up. This has allowed to test the mechanical stability of the mounted modules and investigate system integration aspects such as cabling.

\subsection{Inner Tracker}

The Inner Tracker [6] covers the region of highest particle density closest to the beam-pipe in the three T stations. Though it covers only $1.5 \%$ of the surface area $20 \%$ of the tracks pass through it. To achieve low detector occupancies a fine detector granularity is needed. Therefore, silicon micro-strips with a pitch of $198 \mu$ m were chosen as detector technology. An Inner Tracker station consists of four independent boxes located around the beam-pipe (Fig 4). Each box contains four layers of silicon in the orientation $0^{\circ}, 5^{\circ},-5^{\circ}, 0^{\circ}$. The ladders are mounted on a cooling plate and operated at $5^{\circ} \mathrm{C}$ to reduce leakage currents after several years operation.

Two types of ladder will be used. Above and below the beam-pipe ladders made from a single $11 \mathrm{~cm}$ long sensor will be used. In this case the thickness chosen for sensor is $320 \mu \mathrm{m}$. To the left and right of the beam-pipe $22 \mathrm{~cm}$ long ladders will be used. These are constructed by bonding together two sensors. Testbeam measurements have shown that because of the higher load capacitance it is necessary to use silicon of thickness

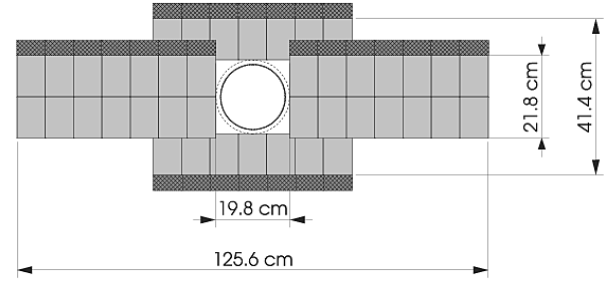

Figure 4. Layout of Station 2 of the Inner Tracker. The darker shading indicates the location of the readout hybrids.

$410 \mu \mathrm{m}$ for these ladders.

The final module production for the Inner Tracker is due to start in August 2005 and is expected to be completed by June 2006 .

\section{EXPECTED PERFORMANCES}

The reconstruction of the trajectories of charged particles in the harsh environment of the LHC is challenging. In each p-p crossing producing a $b \bar{b}$ pair, within the $\mathrm{LHCb}$ acceptance there are $\sim 50$ charged particles. In addition, the amount of material seen by a particle up to the end of the spectrometer amounts to $30-40 \%$ of a radiation length. This leads both to multiple scattering and the generation of secondary particles in hadronic and electromagnetic interactions.

A multi-pass track finding strategy has been developed. In a first pass tracks that traverse the entire spectrometer from the vertex Locator to the last of the $\mathrm{T}$ stations are searched for. These so called 'long tracks' have the highest precision and are the most important for physics analyses. The hits used on these tracks are then flagged as used and a search made for tracks with hits only in the $\mathrm{T}$ stations and the Trigger Tracker. Typically, these tracks are the decay products of $K_{s}$ and hyperon decays occurring outside the acceptance of the Vertex Locator. Finally, with the remaining unused hits a search is made for low momentum particles which are bent out of the acceptance by the magnet but have hits in the 
VeLo and Trigger Tracker.

Figure 5 shows the efficiency to reconstruct long tracks. Above $10 \mathrm{GeV}$ an efficiency of 95\% is found. The ghost rate depends on the transverse momentum of the particle. If it is required that the $p_{t}$ of the reconstructed track is larger than $0.5 \mathrm{GeV}$, as is done in many physics analyses, a ghost rate of $4 \%$ is achieved.

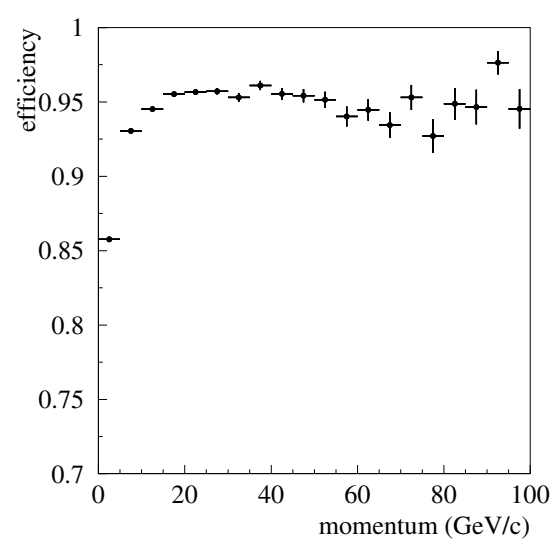

Figure 5. Long track reconstruction efficiency as a function of momentum.

All reconstructed tracks are fitted using a Kalman filter to obtain optimal estimates of the track parameters and the associated covariance matrix. In Fig. 6 the momentum resolution obtained is plotted as a function of the track momentum. It can be seen that the momentum resolution is dominated by the contribution due to multiple scattering up to $\sim 80 \mathrm{GeV}$.

\section{SUMMARY}

The design phase for the subsystems constituting the $\mathrm{LHCb}$ tracking system is now complete. The production of the final detector modules is about to start for the silicon detectors whilst the production of Outer Tracker modules is well advanced. The full detector is expected to be in-

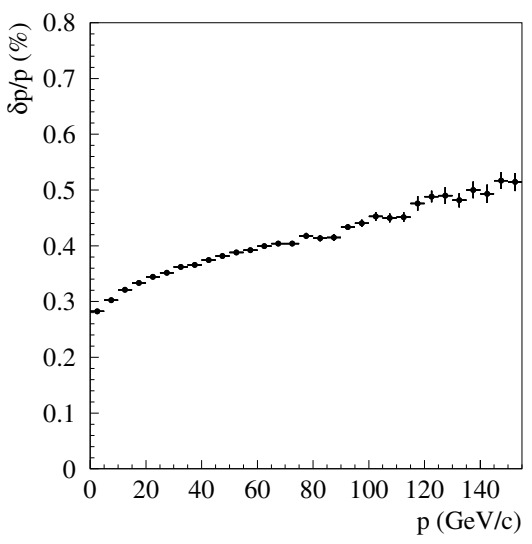

Figure 6. Momentum resolution, $\delta p / p$ for long tracks as a function of momentum, $\mathrm{p}$.

stalled during 2006 and to be ready for first collisions in 2007.

In parallel, track reconstruction algorithms have been developed and shown to give adequate performance on simulated events.

\section{REFERENCES}

1. S. Barsuk. These proceedings.

2. LHCb VELO. Technical Design Report. CERN/LHCC/2001-011.

3. F. Teubert. These proceedings.

4. LHCb Reoptimized Detector Design and Performance. Technical Design Report. CERN/LHCC/2003-030.

5. LHCb Outer Tracker. Technical Design Report. CERN/LHCC/2001-024.

6. LHCb Inner Tracker. Technical Design Report. CERN/LHCC/2002-029. 